\title{
Understanding Eye Tracking Data for Re-engineering Web Pages
}

DOI:

10.1007/978-3-319-04244-2_35

Link to publication record in Manchester Research Explorer

\section{Citation for published version (APA):}

Eraslan, S., Sheng, Q. Z. (Ed.), \& Kjeldskov, J. (Ed.) (2013). Understanding Eye Tracking Data for Re-engineering Web Pages. In Q. Z. Sheng, \& J. Kjeldskov (Eds.), Current Trends in Web Engineering (Vol. 8295, pp. 345-349). (Lecture Notes in Computer Science). Springer Nature. https://doi.org/10.1007/978-3-319-04244-2_35

\section{Published in:}

Current Trends in Web Engineering

\section{Citing this paper}

Please note that where the full-text provided on Manchester Research Explorer is the Author Accepted Manuscript or Proof version this may differ from the final Published version. If citing, it is advised that you check and use the publisher's definitive version.

\section{General rights}

Copyright and moral rights for the publications made accessible in the Research Explorer are retained by the authors and/or other copyright owners and it is a condition of accessing publications that users recognise and abide by the legal requirements associated with these rights.

\section{Takedown policy}

If you believe that this document breaches copyright please refer to the University of Manchester's Takedown Procedures [http://man.ac.uk/04Y6Bo] or contact uml.scholarlycommunications@manchester.ac.uk providing relevant details, so we can investigate your claim.

\section{OPEN ACCESS}




\title{
Understanding Eye Tracking Data for Re-engineering Web Pages
}

\author{
Sukru Eraslan ${ }^{1,2}$, Yeliz Yeşilada ${ }^{1}$, and Simon Harper ${ }^{2}$ \\ 1 Middle East Technical University, Northern Cyprus Campus, Guzelyurt, Mersin 10, Turkey \\ \{seraslan, yyeliz\}@metu.edu.tr \\ 2 University of Manchester, School of Computer Science, United Kingdom \\ sukru.eraslan@postgrad.manchester.ac.uk, \\ simon.harper@manchester.ac.uk
}

\begin{abstract}
Existing re-engineering, namely transcoding, techniques improved disabled and mobile Web users experience by making Web pages more accessible in constrained environments such as on small screen devices and in audio presentation. However, none of these techniques use eye tracking data to transcode Web pages based on understanding and predicting users experience. The overarching goal is to improve the user experience in such constrained environments by using a novel application of eye tracking technology. Thus, this $\mathrm{PhD}$ research project aims to propose an algorithm to identify common scanpaths, which are eye movement sequences, and relating those scanpaths to elements of Web pages. It can then be used to transcode Web pages, for instance, unnecessary information can be removed. It is obvious that both visually disabled and mobile users would benefit from such development.
\end{abstract}

Keywords: eye tracking, scanpaths, commonality, transcoding, re-engineering.

\section{What Is the Setting and History Behind This Project?}

The Web can be accessed by different devices with different requirements and constraints. For example, many people access the Web using their small screen devices while they are mobile and visually disabled people typically access the Web using screen readers. Since Web pages are mainly designed for visual interaction, it is difficult to access them in alternative forms [1]. To address this problem web page transcoding has been proposed. Although existing transcoding techniques improve disabled and mobile Web users experience on the Web [2]3], none of these techniques use eye tracking data to transcode Web pages based on understanding and predicting users' experience.

\section{What Is the Problem to Be Addressed?}

When people access Web pages with their small screen devices, they can experience many difficulties. For example, they may need to scroll a lot which can be annoying and it can be costly to download complex and long pages. Likewise, Web experience can be challenging for visually disabled users. As screen readers follow the source code

Q.Z. Sheng and J. Kjeldskov (Eds.): ICWE 2013 Workshops, LNCS 8295, pp. 345-349, 2013.

(C) Springer International Publishing Switzerland 2013 
of Web pages, visually disabled people have to listen unnecessary clutter to get to the main content. These show that Web pages cannot be accessed properly on small screen devices and in audio presentation. The overarching goal is to improve the user experience in such constrained environments by using a novel application of eye tracking technology. Eye tracking has widely been used to investigate cognitive processes for over 30 years [4], but it is relatively new area in the Web use [56]. While reading, the eyes make quick movements which are called saccades [7]. Between the saccades, the eyes make fixations where they become relatively stationary [7]. Scanpaths are sequences of fixations and saccades on visual stimulus [7]. The objective is to use eye tracking data to generate an algorithm for identifying common scanpaths and relating those scanpaths to elements of Web pages, such that Web pages can be transcoded to improve the user experience. Hence, the following research questions helps to achieve the objective: (1) How can we identify the salient visual elements of Web pages based on aggregated scanpaths? (2) How can we transcode Web pages by using these salient elements of Web pages? (3) How can we quantify efficacy of newly transcoded web pages with regard to mobile and visually disabled users?

\section{What Are Some Current Approaches to This Problem?}

Our literature review has two parts: transcoding and scanpath analysis techniques. Transcoding is a technique used to re-engineer web pages to make them more accessible. Different methods have been used to transcode web pages such as adding a skip link, ranking or reordering or removing irrelevant content [3]. It is observable that they improved disabled and mobile Web user's experience. These methods tended to focus underlying source code of Web pages for visual rendering but they did not concentrate on understanding and predicting users' experience.

Scanpaths on Web pages have been analysed using different methods and algorithms. Most of them use string representations of scanpaths. String representations are created using the sequence of Areas of Interests (AoIs) that gets fixation [8]. Web page AoIs can be generated in different ways such as using grid-layout [8], the source code of Web pages [9] or the fixations' distribution over Web pages [10].

Levenshtein Distance (String-Edit) algorithm has been widely used to analyse scanpaths [5[8]. This algorithm calculates the dissimilarity between scanpaths by transforming the string representation of one scanpath into another one's string representation using a minimum number of operations which are insertion, deletion and substitution. For instance, the dissimilarity between ABCD and ABCE is calculated as 1 (one) by String-Edit algorithm because the transformation can be done by only substituting D with E. The dissimilarities can be used to categorize scanpaths [11]. Also, it can used to investigate differences between the behaviors of people on Web pages [5]. However, this algorithm has considerable weaknesses. Firstly, the substitution costs between all pairs of AoIs may not be the same because their size and distances between AoIs may be different. Substitution cost matrix can be used to store the substitution costs for all pairs of AoIs and then this matrix can be used while calculating the dissimilarity [8]. Needleman and Wunsch algorithm which uses a substitution cost matrix to calculate the similarity between two strings was also used for scanpath analysis [12]. Secondly, String-Edit algorithm does not consider duration metrics, such as fixation duration. However, these 
metrics can be interpreted in different ways. For example, longer fixations can be interpreted as the difficulty for extracting information [7]. ScanMatch method [13] considers fixation duration by defining a particular time duration to cause repetitions of the AoI names in the string representation of the scanpath. For example, if the fixation duration is $200 \mathrm{~ms}$ in AoI A and the particular duration is $100 \mathrm{~ms}$, A is duplicated.

These methods and algorithms have been applied to scanpaths in pair-wise manner but this project aims to find common patterns in a group of scanpaths. One of the techniques used is Transition Matrix which is created using all the scanpaths [11]. In this matrix each cell has the row and column probabilities. Row probabilities allow identifying the next AoI of the particular AoI and column probabilities allow identifying the previous AoI of the particular AoI. When the transition matrix is tried to be used to identify a common scanpath, some significant problems arise: What is the start and end point of the common scanpath? and Which probabilities should be considered?

To address these problems, some other methods can be considered. Shortest Common Subsequence [14] has been mentioned in literature to determine a common scanpath for more than two people but this method has significant weaknesses. For instance, it produces ABDFE as a common scanpath for scanpaths ABE, ADE and AFE. The common scanpath is longer than all of the three scanpaths and it is not supported by the individual scanpaths, for instance, ABE does not include D which is included by the common scanpath.

Some methods, such as T-Pattern [6] and eyePatterns's Discover Patterns [11], try to detect sub-patterns in eye tracking scanpaths. eyePatterns's Discover Patterns [11] have no tolerance to extra items. For instance, ABC can be found as a sub-pattern for $\mathrm{ABC}$ and $\mathrm{ABCD}$ but it cannot be found for $\mathrm{ABC}$ and $\mathrm{ABXCD}$ because of $\mathrm{X}$. This shows its reductionist approach. eyePatterns [11] also has a method to locally align only two sequences to detect sub-patterns. Furthermore, eSeeTrack tool [15] shows the sequence in a time line which allows to see fixation durations. It also considers frequency, so it is able to show which AoIs come after a particular AoI with highlighting the probabilities.

Using multiple sequence alignment was proposed to identify a common scanpath but it was not validated [16]. Also, Dotplots-based algorithm which constructs a hierarchal structure by finding a common scanpath of two sequences with Dotplots algorithm was proposed [17]. The scanpaths are leafs and the common scanpath is the root of the hierarchal structure. In order to eliminate the reductionist approach of the Dotplots algorithm, some statistical methods have been applied.

\section{Why Is This Problem Worth Solving or Worth Solving Better?}

Eye tracking may allow us to drive Web page transcoding by providing a better understand a users' experience and enable the predicting of future interactions. Firstly, it should be understood how sighted users read Web pages on desktop screens. Web pages can then be transcoded to improve the user experience. We assert that both visually disabled and mobile users would benefit from such development. Most mobile operators are also interested in transforming Web pages before they are served to end user 1 , so the results would be beneficial for mobile operators. Moreover, this project will provide

${ }^{1}$ http://www.w3 .org/TR/ct-guidelines / 
benefits for designers, engineers, and practitioners working on Web accessibility and the mobile Web. Since no current approaches consider eye tracking scanpaths, our work will be practical contribution to Human Computer Interaction and Web Science fields.

\section{What Is the Research Methodology to Be Used?}

The programme is split into three work packages (WP). WP1 is related to literature review and testing of the current approaches. Literature Review is essential to understand the existing work in the field. The strengths and weaknesses of these current approaches will be investigated by testing them in the scope of WP1. Eye tracking data will be major material for testing. Existing eye tracking datasets will initially be used [18].

WP2 is the scanpath analysis algorithm development and validation. WP2 consists of two tasks. First task is associated to developing the algorithm. The algorithm will be iteratively developed and tested. Thus, these will be done in parallel. Eye tracking data will be major material for this task, too. Some datasets will be used for the development purpose only and others will be used for the testing purpose to prevent the results being biased. We will start with String-Edit algorithm which has been commonly used in scanpath analysis. Second task is related to experimenting and testing this algorithm. The algorithm will be validated to see whether or not it successfully identifies scanpaths in terms of visual elements. To have different eye tracking dataset for the validation purpose an eye tracking study will be conducted. Thus, this WP concentrates on the first research question.

WP3 focuses on how Web pages can be transcoded by using these salient elements of Web pages and how we can quantify efficacy of newly transcoded web pages with regard to mobile and visually disabled users. Current transcoding techniques will be investigated to see how they can be used with eye tracking data to transcode web pages based on understanding and predicting users experience. Hence, this WP is related to the second and third research questions.

\section{What Is the Agenda Ahead?}

As this is a three-year $\mathrm{PhD}$ project, the work packages (WP) are distributed over thirtysix months as shown in Fig. 1] where dark gray shows main tasks and light gray shows background tasks. WP1 (Literature Review and Testing of Current Approaches) will be continued until the end of this project to see whether or not relevant works have been done. WP2 (Algorithm Development and Validation) will be started after eight months. The algorithm will be developed by considering the strengths and weaknesses of the

\begin{tabular}{|c|c|c|c|c|c|c|c|c|c|c|c|c|c|c|c|c|c|c|c|c|c|c|c|c|c|c|c|c|c|c|c|c|c|c|c|c|}
\hline \multirow{2}{*}{\multicolumn{2}{|c|}{$\begin{array}{c}\text { Work Packages } \\
\text { (WP) }\end{array}$}} & \multicolumn{35}{|c|}{ Months } \\
\hline & & 1 & 2 & 3 & 4 & 5 & 6 & 7 & 8 & 9 & 10 & 111 & \begin{tabular}{l|l}
12 & 1 \\
\end{tabular} & \begin{tabular}{l|l}
13 & 1 \\
1
\end{tabular} & \begin{tabular}{l|l}
14 & 1 \\
\end{tabular} & \begin{tabular}{l|l}
5 & 1 \\
\end{tabular} & \begin{tabular}{l|l}
6 & 17 \\
\end{tabular} & \begin{tabular}{l|l|}
7 & 18 \\
\end{tabular} & 19 & 20 & 21 & 22 & 23 & 24 & \begin{tabular}{|l|l|}
25 & 2 \\
\end{tabular} & 26 & 27 & 28 & 29 & 30 & 31 & 32 & 33 & 34 & \begin{tabular}{l|l}
35 & 3 \\
\end{tabular} & 36 \\
\hline \multicolumn{37}{|c|}{ 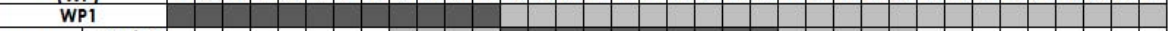 } \\
\hline \multirow{2}{*}{ WP2 } & Task 1 & & & & & & & & & & & & & & & & & & & & & & & & & & & & & & & & & & & \\
\hline & Task 2 & & & & & & & & & & & & & & & & & & & & & & & & & & & & & & & & & & & \\
\hline \multicolumn{2}{|c|}{ WP3 } & & & & & & & & & & & & & & & & & & & & & & & & & & & & & & & & & & & \\
\hline
\end{tabular}

Fig. 1. Agenda 
current approaches (Task 1) while testing the current approaches. Algorithm Validation stage (Task 2) will be started before the completion of algorithm because some weaknesses can be recognised at this stage. Eight months are allocated for WP3 which is related to transcoding because this project's supervisors have significant knowledge about transcoding [32]. This project has started in September 2012 and therefore we are currently working on WP1 and about the start to WP2.

\section{References}

1. Harper, S., Yesilada, Y.: Web accessibility and guidelines. In: Web Accessibility: A Foundation for Research, 1st edn. Human-Computer Interaction Series, ch. 6, pp. 61-78. Springer, London (2008)

2. Yesilada, Y., Stevens, R., Harper, S., Goble, C.: Evaluating dante: Semantic transcoding for visually disabled users. ACM Trans. Comput.-Hum. Interact. 14(3), 14 (2007)

3. Asakawa, C., Takagi, H.: Transcoding. In: Harper, S., Yesilada, Y. (eds.) Web Accessibility: A Foundation for Research. Human-Computer Interaction Series, pp. 231-260. Springer (2008)

4. Rayner, K.: Eye movements in reading and information processing: 20 years of research. Psychological Bulletin 124, 372-422 (1998)

5. Josephson, S., Holmes, M.E.: Visual attention to repeated internet images: testing the scanpath theory on the world wide web. In: ETRA 2002, pp. 43-49. ACM, NY (2002)

6. Mast, M., Burmeister, M.: Exposing repetitive scanning in eye movement sequences with t-pattern detection. In: IADIS IHCI 2011, Rome, Italy, pp. 137-145 (2011)

7. Poole, A., Ball, L.J.: Eye tracking in human-computer interaction and usability research: Current status and future. In: Ghaoui, C. (ed.) Prospects. Encyclopedia of Human-Computer Interaction. Idea Group, Inc., Pennsylvania (2005)

8. Takeuchi, H., Habuchi, Y.: A quantitative method for analyzing scan path data obtained by eye tracker. In: CIDM 2007, March 1-April 5, pp. 283-286 (2007)

9. Akpinar, E., Yesilada, Y.: Vision based page segmentation: Extended and improved algorithm. Technical report, Middle East Technical University Northern Cyprus Campus (2012)

10. Santella, A., DeCarlo, D.: Robust clustering of eye movement recordings for quantification of visual interest. In: ETRA 2004, pp. 27-34. ACM, New York (2004)

11. West, J.M., Haake, A.R., Rozanski, E.P., Karn, K.S.: Eyepatterns: software for identifying patterns and similarities across fixation sequences. In: ETRA 2006, pp. 149-154. ACM, NY (2006)

12. Day, R.F.: Examining the validity of the needleman\&wunsch algorithm in identifying decision strategy with eye-movement data. Decision Support Systems 49(4), 396-403 (2010)

13. Cristino, F., Mathot, S., Theeuwes, J., Gilchrist, I.D.: Scanmatch: a novel method for comparing fixation sequences. Behav. Res. Methods 42(3), 692-700 (2010)

14. Räihä, K.-J.: Some applications of string algorithms in human-computer interaction. In: Elomaa, T., Mannila, H., Orponen, P. (eds.) Ukkonen Festschrift 2010. LNCS, vol. 6060, pp. 196-209. Springer, Heidelberg (2010)

15. Tsang, H.Y., Tory, M., Swindells, C.: eseetrack: Visualizing sequential fixation patterns. IEEE Transactions on Visualization and Computer Graphics 16(6), 953-962 (2010)

16. Hembrooke, H., Feusner, M., Gay, G.: Averaging scan patterns and what they can tell us. In: ETRA 2006, p. 41. ACM, New York (2006)

17. Goldberg, J.H., Helfman, J.I.: Scanpath clustering and aggregation. In: ETRA 2010, pp. 227234. ACM, NY (2010)

18. Brown, A., Jay, C., Harper, S.: Tailored presentation of dynamic web content for audio browsers. International Journal of Human-Computer Studies 70(3), 179-196 (2012) 